\title{
Astrophysics of Reference Frame Tie Objects
}

\author{
Principal Investigator: Kenneth J. Johnston, (USNO) \\ Team Members: \\ David Boboltz (USNO), Alan Lee Fey (USNO), Ralph A. Gaume (USNO), \\ Norbert Zacharias (USNO).
}

The Astrophysics of Reference Frame Tie Objects Key Science program will investigate the underlying physics of SIM grid objects. Extragalactic objects in the SIM grid will be used to tie the SIM reference frame to the quasi-inertial reference frame defined by extragalactic objects and to remove any residual frame rotation with respect to the extragalactic frame. The current realization of the extragalactic frame is the International Celestial Reference Frame (ICRF). The ICRF is defined by the radio positions of 212 extragalactic objects and is the IAU sanctioned fundamental astronomical reference frame. This key project will advance our knowledge of the physics of the objects which will make up the SIM grid, such as quasars and chromospherically active stars, and relates directly to the stability of the SIM reference frame. The following questions concerning the physics of reference frame tie objects will be investigated.

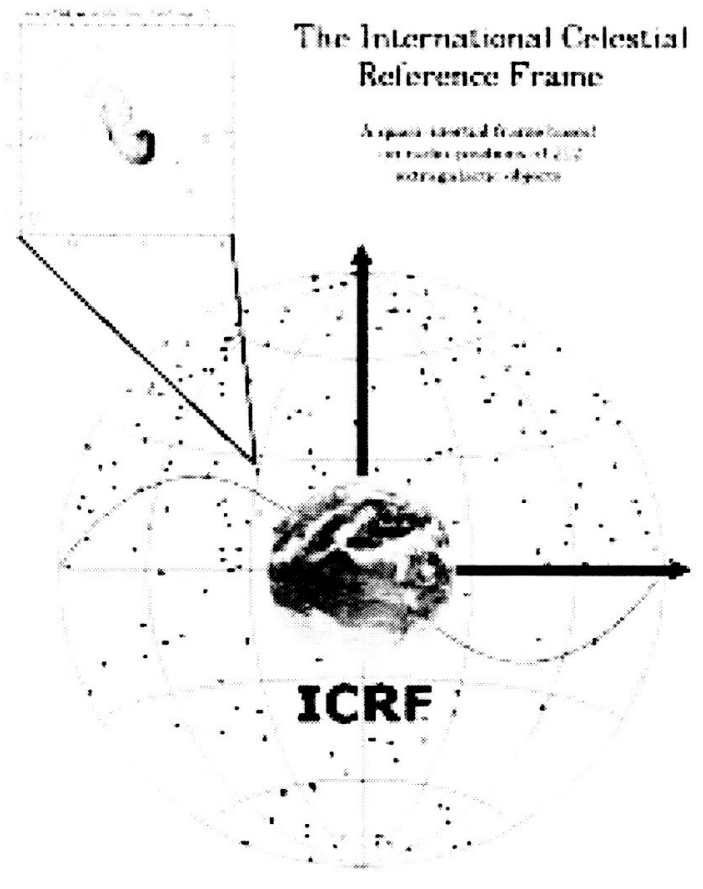

Figure 1: Figure shows a schematic representation of the ICRF as a projection of extragalactic radio sources onto the celestial sphere. The inset shows the $8.55 \mathrm{GHz}$ radio frequency image of a representative source.

What is the origin of optical emission in quasars? In the standard theory of extragalactic radio sources, emission from quasars and active galactic nuclei (AGN) is assumed to be powered by a central engine (presumably a black hole) where energetic phenomena occur. The origin of the optical wavelength radiation from AGN identified with compact radio sources is not well 
established. There are several possibilities: 1) the optical radiation is thermal cmission from an accretion disk; 2) the optical radiation is non-thermal emission from a magnetized corona or wind emanating from the central region of the accretion disk; or 3) the optical radiation is emission from a relativistic jet beamed toward the observer. SIM observations will allow a direct test of this model.

Are the optical photo-centers of quasars compact and positionally stable on the microarcsecond level? It is well known that variable intrinsic structure of AGN at radio wavelengths has a significant impact on (degrades) radio positional accuracy. Currently, there is very little available information on whether the optical counterparts of the radio objects are compact at the level of astrometric precision expected from SIM. Although the core of a quasar's optical emission may originate in a region as small as $1 \mathrm{pc}(200$ microarcseconds at $1 \mathrm{Gpc})$, some degree of photocenter wander should be expected, probably correlated with optical variability. Motion of a quasar's photocenter may also result from a variable nucleus in combination with effects in the larger (albeit fainter) host galaxy.

Are binary black hole mergers responsible for quasars? Optical photocenter wander of quasars can be used to search for binary black hole signatures and thus test the hypothesis that binary black hole mergers are responsible for most quasars. A positive detection of binary black hole induced motions in the optical photocenter of quasars would be good evidence to support this hypothesis.

What is(are) the emission mechanism(s) responsible for generating radio emission in chromospherically active stars. Is the emission thermal, relativistic synchrotron or gyro-synchrotron? No consensus has yet developed concerning the physics of the formation and evolution of the radio emission associated with the active binary star systems. Also, for most active binaries, the location of the radio emission with respect to the binary components is unknown; e.g. is the radio emitting region centered on one of the stars, is it located in the intra-binary region, or does it surround both stars? This uncertainty can be attributed, in part, to inadequacies in the radio/optical frame link.

What causes the transition of spherically symmetric Asymptotic Giant Branch (AGB) stars to asymmetric planetary nebulae (PNe)? Models based on interacting winds have had considerable success explaining PNe morphologies. However, it is still unknown whether the asymmetries observed in $\mathrm{PNe}$ are present in the progenitor AGB stars themselves, and whether these asymmetries could be produced by mechanisms such as non-radial pulsations or unseen companions. SIM observations of a number of AGB stars shown to have asymmetric envelopes, through radio observations of circumstellar masers, will allow a test of these theories. 


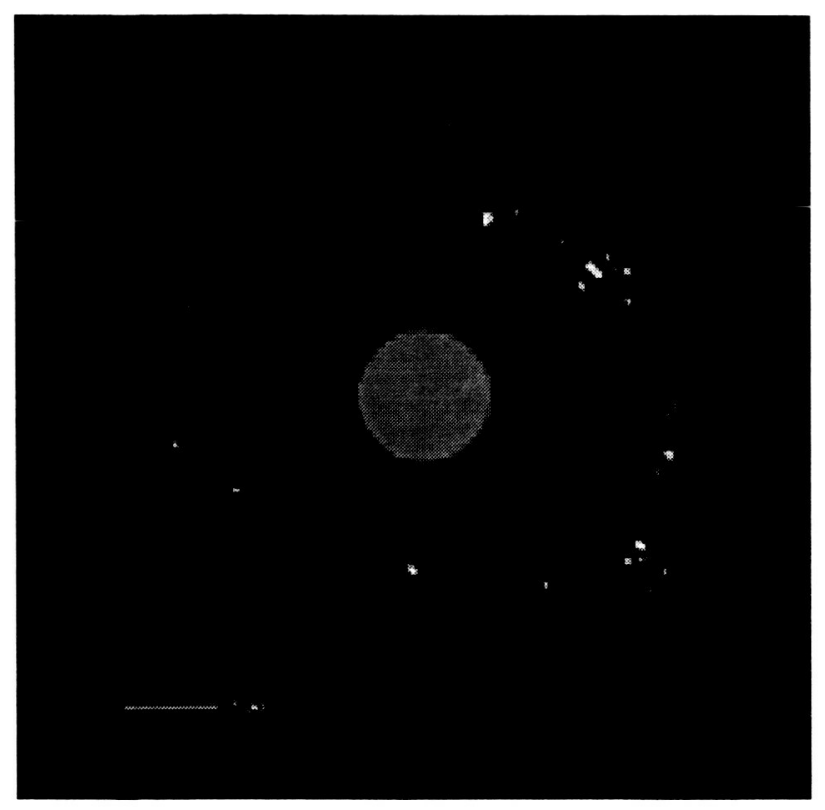

Figure 2: Figure shows the asymmetrical $\mathrm{SiO}$ maser shell toward the Mira variable IK Tauri with nearly a 2:1 axial ratio. The location of the stellar disk (red circle) relative to the masers is pure conjecture. 